\title{
Extensive-Form Games and Strategic Complementarities
}

\author{
Federico Echenique*
}

September 27, 2000

\begin{abstract}
I prove the subgame-perfect equivalent of the basic result for Nash equilibria in normal-form games of strategic complements: the set of subgame-perfect equilibria is a non-empty, complete lattice. For this purpose I introduce a device that allows the study of the set of subgame-perfect equilibria as the set of fixed points of a correspondence. The correspondence has a natural interpretation. My results are limited because extensive-form games of strategic complementarities turn outsurprisingly - to be a very restrictive class of games.
\end{abstract}

JEL Classification: C72, C73.

*Department of Economics, University of California at Berkeley and Departamento de Economía, Facultad de Ciencias Sociales. email: fede@econ.berkeley.edu. I am very grateful to Ilya Segal and Chris Shannon for many helpful discussions. I also wish to thank Bob Anderson, and Matthew Rabin for their comments. The usual disclaimer applies. 


\section{Introduction}

In this paper I define extensive-form games of strategic complementarities and prove the subgame-perfect equivalent of the basic result for Nash equilibria in normal-form games of strategic complements: the set of subgame-perfect Nash equilibria (SPNE) is a nonempty, complete lattice. This has strong implications; not only does it give a general existence proof, it also allows the use of comparative statics techniques. ${ }^{1}$ While this seems to be a promising result, I also show that, in extensive-form games, the assumption of strategic complementarities is - surprisingly — very restrictive.

Equilibria are usually analyzed by means of fixed-point methods. This has not been the case for SPNE. A methodological contribution of this paper is the introduction of a device, the "extended best-response correspondence", with the property that the set of SPNE of a game coincides with the set of fixed points of the extended best-response correspondence. The model of extensive-form games that I use allows time to be continuous, so the extended best-response correspondence can also be used to analyze SPNE of continuous-time games.

Existence of SPNE in finite games (finite number of players, of actions, and of stages) follows from Zermelo's Theorem. Harris, Reny, and Robson (1995) present an example of a game without an SPNE that is a two-stage game with a finite number of players and where only one player has an infinite strategy space. Hence, existence of SPNE is not guaranteed after a minimal departure from the hypothesis of Zermelo's Theorem.

Here I show that the existence of SPNE follows from strategic complementarities; concretely, that the set of SPNE of an game whose normal-form is a game of strategic complementarities, is a non-empty, complete lattice.

Proofs of existence of SPNE in non-finite games are provided by Harris, Reny, and Robson (1995) for games of almost-perfect information where a public randomization device is present, by Harris (1985b), Harris (1985a) and Hellwig and Leininger (1987) for

\footnotetext{
${ }^{1}$ It can be shown that monotone comparative statics techniques would, among other things, eliminate the need to focus on Markov-Perfect equilibria in many dynamic oligopoly models. I chose not to stress this consequence of my results because, as will become clear, it has a very limited applicability.
} 


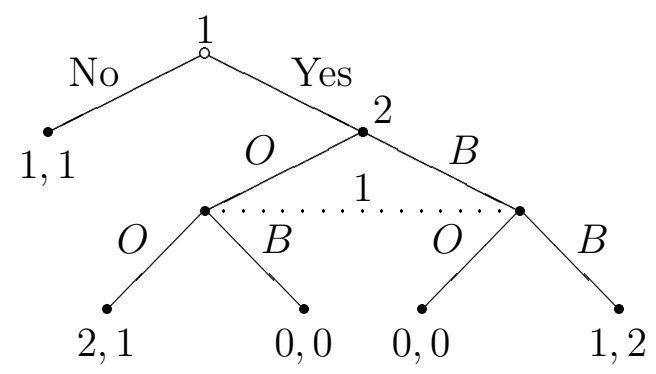

Figure 1: Optional Battle of the Sexes

games of perfect information; and by Fudenberg and Levine (1983) for classes of games with strong "continuity at infinity" properties.

My results apply to continuous-time games and to games of imperfect information that are not necessarily games of almost-perfect information. I am not aware of any other existence result that applies to continuous-time games. I do not impose any structure on games beyond complementarities and conventional topological assumptions - the important problem with my results is that the assumption of complementarities is very strong.

Games of strategic complementarities were first studied by Topkis (1979) and introduced into economics by Vives (1990). There are many examples of economic models that are games of strategic complements (see Milgrom and Roberts (1990), Topkis (1998), and Vives (1999)). By now it is fair to say that complementarities in normal-form games is a very useful and common structure. Here I show that, while still very useful, complementarities in dynamic contexts are rare.

To illustrate the problem, consider the game in Figure 1. This is "Optional Battle of the Sexes". Here, player One chooses first to say Yes or No. If One says No then payoffs are 1 each. If One says Yes then they play a Battle of the Sexes game: they simultaneously choose an element of $\{O, B\}$. If the choice is $(O, O)$ then player One gets 2 while Two gets 1 . If they choose $(O, B)$ or $(B, O)$ then both get a payoff of 0 . If the outcome is $(B, B)$ then payoffs are $(1,2)$

It is easy to see that Battle of the Sexes (BoS, the simultaneous-move game that follows after One chooses Yes) is a game of strategic complementarities. Player One's 
best response to Two playing $B$ is to play $B$ and One's response to Two playing $O$ is to play $O$. So, a change by Two from $B$ to $O$ makes One change in the same direction. This is also true for player Two: a change by One from $B$ to $O$ makes Two change in the same direction. Imposing an order on the players' strategies, we can say that $O$ is "larger" than $B$. Then the best response of each player is increasing in the other player's choice of strategy, this is the crucial property of a game of strategic complementarities (indeed it is easily seen that BoS satisfies the definition of a game of strategic complementarities in e.g. Milgrom and Roberts (1990)).

Now, consider the extensive-form game Optional BoS and let us impose an order on the set $\{$ No, Yes $\}$. Let the action "Yes" at One's initial decision node be larger than "No". Then the strategy No-O (say No at the initial node and plan to play $O$ in Battle of the Sexes) is smaller than Yes- $O$ and No- $B$ is smaller than Yes- $B$. But, when One is playing No- $B$ it is optimal for Two to play $O$, while if we increase One's strategy to Yes- $B$ then it is uniquely optimal for Two to play $B$. This implies that Two's strategy is not increasing in One's strategy choice. We could try to fix this by saying that $B$ is larger than $O$, but then the problem would arise when One increases the strategy from No-O to Yes-O.

It turns out that it is possible to make Optional BoS a game of strategic complementarities. The solution is to say that the action Yes is smaller than No. This shows that extensive-form games of strategic complementarities are not trivial. ${ }^{2}$ But unfortunately the simple solution in Optional BoS is not feasible in general. I shall show how a complication of Optional BoS yields a game that cannot be transformed into a game of strategic complementarities. I will argue also that most dynamic games of economic interest cannot be transformed into games of strategic complementarities.

Section 2 presents definitions and notation. Section 3 introduces the extended bestresponse correspondence and the main results of the paper. Section 4 show how complementarities are a restrictive assumption by discussing some examples.

\footnotetext{
${ }^{2}$ It is not true that they must be dominance-solvable, as might be suggested by the discussion above.
} 


\section{Generalized Extensive-Form Games}

\subsection{Basic Definitions and Notation}

A detailed discussion of the concepts defined in this subsection is in Topkis (1998). A set $X$ with a transitive, reflexive, antisymmetric binary relation $\preceq$ is a lattice if whenever $x, y \in X$, both $x \wedge y=\inf \{x, y\}$ and $x \vee y=\sup \{x, y\}$ exist in $X$. It is complete if for every nonempty subset $A$ of $X, \inf A, \sup A$ exist in $X$. For two subsets $A, B$ of $X$, say that $A$ is smaller than $B$ in the strong set order if $a \in A, b \in B$ implies $a \wedge b \in A, a \vee b \in B$. The order-interval topology on a lattice is obtained by taking the closed intervals $[x, y]=\{z \in X: x \preceq z \preceq y\}$ as a sub-basis for the closed sets. All lattices in the paper will be endowed with the order-interval topology. All products of partially ordered sets are endowed with the product order. All products of topological spaces are endowed with the product topology.

If $X$ is a lattice, a function $f: X \rightarrow \mathbf{R}$ is quasisupermodular if for any $x, y \in X$, $f(x) \geq f(x \wedge y)$ implies $f(x \vee y) \geq f(y)$ and $f(x)>f(x \wedge y)$ implies $f(x \vee y)>f(y){ }^{3}$ Let $T$ be a partially ordered set. A function $f: X \times T \rightarrow \mathbf{R}$ satisfies the singlecrossing condition in $(x, t)$ if whenever $x \prec x^{\prime}$ and $t \prec t^{\prime}, f(x, t) \leq f\left(x^{\prime}, t\right)$ implies that $f\left(x, t^{\prime}\right) \leq f\left(x^{\prime}, t^{\prime}\right)$ and $f(x, t)<f\left(x^{\prime}, t\right)$ implies that $f\left(x, t^{\prime}\right)<f\left(x^{\prime}, t^{\prime}\right)$. The restriction of a function $f: X \rightarrow Y$ to a subset $X^{\prime} \subseteq X$ is denoted $\left.f\right|_{X^{\prime}}$.

\subsection{Definition of Generalized Extensive-Form Games}

I present a definition of extensive-form games that has information sets, as opposed to decision nodes, as primitives. It is really only a slight variation on the usual rules for drawing game trees, but it results in a considerably more general framework because it allows time to be continuous and does not impose perfect recall or partitioned information structures. ${ }^{4}$ I hope that the benefits of having results that apply to continuous-time games are important enough to balance the cost of a slightly unfamiliar framework.

\footnotetext{
${ }^{3}$ Quasisupermodularity is an ordinal notion of complementarities, it was introduced by Milgrom and Shannon (1994).

${ }^{4}$ See Fudenberg and Tirole (1991) and Osborne and Rubinstein (1994) for the two usual definitions.
} 


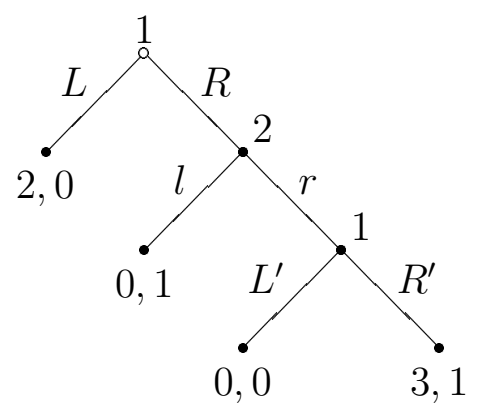

Figure 2: An extensive-form game of strategic complementarities

Besides its generality, this model of extensive-form games is more parsimonious than the usual one, therefore the proofs are easier and sharper than they would be otherwise.

I shall use the simple game in Figure 2 to illustrate the concepts as they are introduced. The game can be described as follows. First, player One selects an element in $\{L, R\}$. If she selects $L$ then the game "ends" and the payoffs are 2 to player One and 0 to player Two. If she selects $R$ then Two gets to choose between $l$ and $r$. If he chooses $l$ then she gets 1 while One gets 0 . If he chooses $r$ then player One gets to choose an element in $\left\{L^{\prime}, R^{\prime}\right\}$. Payoffs are $(0,0)$ and $(3,1)$ after One chooses $L^{\prime}$ and $R^{\prime}$, respectively. Let $a_{1}$ be the first node at which player One moves, $a_{2}$ be the second node at which she moves and $b$ be the node at which player Two moves.

A generalized extensive-form game will be described as follows. Let $N=\{1, \ldots n\}$ be the set of players. Let $H$ be a set; the elements of $H$ will be referred to as "information sets". Let $\mathcal{H}=\left\{H_{\alpha}: \alpha \in I\right\}$ be a collection of subsets of $H$ and $\left\{H^{i}: i \in N\right\}$ a partition of $H$. The interpretation will be that player $i$ is endowed with a collection $H^{i}$ of information sets and that the elements of $H=\cup_{i=1}^{n} H^{i}$ are the information sets of the game. For each $\alpha \in I, H_{\alpha} \subseteq H$ should be interpreted as a "subgame" of $H$ (this interpretation is made precise below).

In the example in Figure 2, $N=\{1,2\}, H=\left\{a_{1}, a_{2}, b\right\}, H^{1}=\left\{a_{1}, a_{2}\right\}$ and $H^{2}=\{b\}$. There are three subgames in the example, let $I=\left\{\alpha_{0}, \alpha_{1}, \alpha_{2}\right\}, H_{\alpha_{0}}=\left\{a_{1}, a_{2}, b\right\}, H_{\alpha_{1}}=$ $\left\{a_{2}, b\right\}$, and $H_{\alpha_{2}}=\left\{a_{2}\right\} ; \mathcal{H}=\left\{H_{\alpha_{0}}, H_{\alpha_{1}}, H_{\alpha_{2}}\right\}$.

I make two assumptions about $\mathcal{H}$, the collection of "subgames". First, that $H$ itself belongs to this class of subgames. Let $\alpha_{0} \in I$ satisfy $H=H_{\alpha_{0}}$. Second, if $\left\{H_{\alpha}: \alpha \in I^{\prime}\right\} \subseteq$ 
$\mathcal{H}$ is a subcollection of $\mathcal{H}$ such that any $\alpha, \alpha^{\prime} \in I^{\prime}$ satisfy either $H_{\alpha} \subseteq H_{\alpha^{\prime}}$ or $H_{\alpha^{\prime}} \subseteq H_{\alpha}$, then $\cup_{\alpha \in I^{\prime}} H_{\alpha} \in \mathcal{H}$. That is, I assume that $\mathcal{H}$ is closed under increasing unions. For every $\alpha \in I,\left\{H^{i}: i \in N\right\}$ induces a partition on $H_{\alpha}$ : let $H_{\alpha}^{i}=H_{\alpha} \cap H^{i}$ be the set of player $i$ 's information sets in subgame $H_{\alpha}$. Note that $\mathcal{H}^{i}=\left\{H_{\alpha}^{i}: \alpha \in I\right\}$ is also closed under increasing unions. It is easy to verify that the example in Figure 2 satisfies these assumptions, as does any well-defined game tree.

The players choose actions at each of their information sets. For each $h \in H$, let $A(h)$ be the set of actions available to the player that moves at information set $h$. Each $A(h)$ is endowed with a Hausdorff topology. The set of all possible actions is denoted by $\mathcal{A}=\cup_{h \in H} A(h)$. Player $i$ 's strategy space in subgame $\alpha \in I$ is $S_{\alpha}^{i}=$ $\left\{s: H_{\alpha}^{i} \rightarrow \mathcal{A}: s(h) \in A(h)\right.$ for all $\left.h \in H_{\alpha}^{i}\right\}=\Pi_{h \in H_{\alpha}} A(h)$. Let $S_{\alpha}=\times_{i=1}^{n} S_{\alpha}^{i}$. Each player is endowed with preferences over strategy profiles in subgame $\alpha \in I$. These preferences are represented by a collection of payoff functions $u_{\alpha}^{i}: S_{\alpha} \rightarrow \mathbf{R}$.

In the example, $A\left(a_{1}\right)=\{L, R\}, A(b)=\{l, r\}$ and $A\left(a_{2}\right)=\left\{L^{\prime}, R^{\prime}\right\}$, so that $\mathcal{A}=\left\{L, L^{\prime}, R, R^{\prime}, l, r\right\}$. The strategy space for player 1 for the whole game is $S_{\alpha_{0}}^{1}=$ $\left\{L L^{\prime}, L R^{\prime}, R L^{\prime}, R R^{\prime}\right\}$-where $L L^{\prime}$ means that 1 plans to play $L$ at her first decision node, $a_{1}$, and then $L^{\prime}$ at her second decision node, $a_{2}$, and so on. The strategy space for player 2 for the whole game is simply $S_{\alpha_{0}}^{2}=\{l, r\}$. The strategy spaces for the other subgames are $S_{\alpha_{1}}^{1}=\left\{L^{\prime}, R^{\prime}\right\}, S_{\alpha_{1}}^{2}=\{l, r\}, S_{\alpha_{2}}^{1}=\left\{L^{\prime}, R^{\prime}\right\}$ and $S_{\alpha_{2}}^{2}=\{\emptyset\}$. The choice of $\emptyset$ for player 2 in subgame $\alpha_{2}$ formalizes that only 1 makes a choice in this subgame. The players' preferences in each subgame are immediate from Figure 2, $u_{\alpha_{0}}^{1}\left(L L^{\prime}, l\right)=2, u_{\alpha_{0}}^{2}\left(L L^{\prime}, l\right)=0$, $u_{\alpha_{1}}^{1}\left(L^{\prime}, l\right)=0, u_{\alpha_{1}}^{2}\left(L^{\prime}, l\right)=1, u_{\alpha_{2}}^{1}\left(L^{\prime}, \emptyset\right)=0, u_{\alpha_{2}}^{2}\left(L^{\prime}, \emptyset\right)=0$, etc.

Definition 1 A collection of payoff functions $\left\{u_{i}^{\alpha}: i \in N, \alpha \in I\right\}$ is consistent if, for every $i \in N$ and $\alpha, \alpha^{\prime} \in I$, whenever $H_{\alpha^{\prime}} \subseteq H_{\alpha}, s_{\alpha}^{-i} \in S_{\alpha}^{-i}, s_{\alpha}^{i}, \tilde{s}_{\alpha}^{i} \in S_{\alpha}^{i}$ and $z_{\alpha^{\prime}}^{i} \in$ $S_{\alpha^{\prime}}^{i}$, the inequalities $u_{\alpha}^{i}\left(\tilde{s}_{\alpha}^{i}, s_{\alpha}^{-i}\right) \leq u_{\alpha}^{i}\left(s_{\alpha}^{i}, s_{\alpha}^{-i}\right)$ and $u_{\alpha^{\prime}}^{i}\left(\left.s_{\alpha}^{i}\right|_{H_{\alpha^{\prime}}^{i}},\left.s_{\alpha}^{-i}\right|_{H_{\alpha^{\prime}}^{-i}}\right) \leq u_{\alpha^{\prime}}^{i}\left(z_{\alpha^{\prime}}^{i},\left.s_{\alpha}^{-i}\right|_{H_{\alpha^{\prime}}^{-i}}\right)$ imply that $u_{\alpha}^{i}\left(\tilde{s}_{\alpha}^{i}, s_{\alpha}^{-i}\right) \leq u_{\alpha}^{i}\left(\left.s_{\alpha}^{i}\right|_{H_{\alpha}^{i} \backslash H_{\alpha^{\prime}}^{i}}, z_{\alpha^{\prime}}^{i}, s_{\alpha}^{-i}\right)$. The collection of payoff functions satisfies continuity if, for all $\alpha \in I, i \in N$, and $s_{\alpha}^{-i} \in S_{\alpha}^{-i}, s_{\alpha}^{i} \mapsto u_{\alpha}^{i}\left(s_{\alpha}^{i}, s_{\alpha}^{-i}\right)$ is an upper semicontinuous function. 
Payoffs are consistent if, given opponents' strategy $s^{-i}$, whenever $s_{\alpha}^{i}$ performs better than $\tilde{s}_{\alpha}^{i}$ in subgame $H_{\alpha} \supset H_{\alpha^{\prime}}$ and $z_{\alpha^{\prime}}^{i}$ performs better than $s_{\alpha}^{i}$ in subgame $H_{\alpha^{\prime}}$, it must be that the combined strategy that follows $s_{\alpha}^{i}$ in $H_{\alpha}^{i} \backslash H_{\alpha^{\prime}}^{i}$ and follows $z_{\alpha^{\prime}}^{i}$ in $H_{\alpha^{\prime}}^{i}$, cannot perform worse than $\tilde{s}_{\alpha}^{i}$.

The payoffs in the example are consistent: Fix the strategy $s_{\alpha_{0}}^{2}=l$ by player 2 in subgame $\alpha_{0}$, the "whole" game. Given any strategy $s_{\alpha_{0}}^{1}$, player 1's payoff is independent of choices in node $a_{2}$. In particular, choosing $R^{\prime}$, the dominant strategy in subgame $\alpha_{2}$, does not decrease the payoff to following $s_{\alpha_{0}}^{1}$. Now, consider $s_{\alpha_{0}}^{2}=r$. The only case where the requirement consistency has bite is for the strategy $L L^{\prime}$. In subgame $\alpha_{0}, L L^{\prime}$ is preferred by 1 to $R L^{\prime}$. But, in subgame $\alpha_{2}, R^{\prime}$ is better than $L^{\prime}$. Consistency then requires that $L R^{\prime}$ be preferred to $R L^{\prime}$ in subgame $\alpha_{0}$-which is satisfied by the specified payoffs.

The example illustrates why payoffs in any well-defined game tree are consistent. Given $i$ 's strategy $s_{\alpha}^{i}$ and opponents' strategies $s_{\alpha}^{-i}$, if a subgame $\alpha^{\prime}$ is not reached then $i$ is indifferent among her choices in this subgame and she cannot do worse by picking something that is better in the subgame. On the other hand, if subgame $\alpha^{\prime}$ is reached then payoffs will be given by choices in $\alpha^{\prime}$. Choosing a better strategy in subgame $\alpha^{\prime}$ can only improve the payoff to $s_{\alpha}^{i}$.

The definition of a generalized extensive-form game is complicated enough to warrant an enumeration of its components:

Definition 2 The sextuple $\Gamma=\left\{N, H,\left\{H_{\alpha}: \alpha \in I\right\},\left\{H^{i}: i \in N\right\},\{A(h): h \in H\}\right.$, $\left.\left\{u_{\alpha}^{i}: i \in N, \alpha \in I\right\}\right\}$ is a generalized extensive-form game if:

- $N=\{1,2, \ldots n\}$ is the set of players;

- $H$ is a set of information sets;

- $\left\{H^{i}: i \in N\right\}$ is a partition of $H$;

- $\left\{H_{\alpha}: \alpha \in I\right\}$ is a collection of subsets of $H$ that is closed under increasing unions and such that $H_{\alpha_{0}}=H$ for some $\alpha_{0} \in I$; 
- $\{A(h): h \in H\}$ is a collection of action sets, each endowed with a Hausdorff topology and compact;

- $\left\{u_{i}^{\alpha}: i \in N, \alpha \in I\right\}$ is a collection of consistent payoff functions that satisfies continuity.

For any subgame $\alpha \in I, \Gamma$ induces naturally an extensive-form game $\Gamma_{\alpha}$ : let $H_{\alpha}$ be the set of information sets of $\Gamma_{\alpha}$, let the subgames $\gamma \in I$ with $H_{\gamma} \subseteq H_{\alpha}$ be the subgames of $\Gamma_{\alpha}$; and let action sets and payoffs be defined as in $\Gamma$. I will use "subgame" to denote both the set $H_{\alpha}$ and its corresponding extensive-form game $\Gamma_{\alpha}$.

A strategy profile $s_{\alpha}$ is a subgame-perfect Nash equilibrium (SPNE) in subgame $\alpha \in I$ if, for every $\gamma \in I$ such that $H_{\gamma} \subseteq H_{\alpha}$ and every $i \in N$,

$$
\left.s_{\alpha}^{i}\right|_{H_{\gamma}} \in \operatorname{argmax}_{\tilde{s}_{\gamma}^{i} \in S_{\gamma}^{i}} u^{\gamma}\left(\tilde{s}_{\gamma}^{i},\left.s_{\alpha}^{-i}\right|_{H_{\gamma}}\right) .
$$

I shall refer to the SPNE in subgame $\alpha_{0}$, the whole game, as simply SPNE. Note that a strategy profile is a SPNE if and only if its restriction to any subgame is a SPNE in that subgame.

Definition 3 A generalized extensive-form game $\Gamma$ is an extensive-form game of strategic complementarities if $A(h)$ is a complete lattice for all $h \in H$ and if, for all $\alpha \in I$ and $i \in N, s_{\alpha}^{i} \mapsto u_{\alpha}^{i}\left(s_{\alpha}^{i}, s_{\alpha}^{-i}\right)$ is quasisupermodular on $S_{\alpha}^{i}$, and $u_{\alpha}^{i}$ satisfies the single-crossing condition in $\left(s_{\alpha}^{i}, s_{\alpha}^{-i}\right)$.

It is slightly cumbersome to show that the game in Figure 1, has strategic complements. I leave this for section 4 .

\subsection{Examples of Generalized Extensive-Form Games}

\subsubsection{Optional BoS}

I shall present the current notation for the "Optional Battle of the Sexes" game from the introduction. Let $a_{1}$ be the initial node, $b$ be the node at which player Two moves and $a_{2}$ be One's information set after that Two has moved. Then, $H=\left\{a_{1}, a_{2}, b\right\}$, 
$H^{1}=\left\{a_{1}, a_{2}\right\}, H^{2}=\{b\}$. There are two subgames, so $I=\left\{\alpha_{0}, \alpha_{1}\right\}, H_{\alpha_{0}}=\left\{a_{1}, a_{2}, b\right\}$ and $H_{\alpha_{1}}=\left\{b, a_{2}\right\}$. Action spaces are $A\left(a_{1}\right)=\{$ Yes, No $\}, A(b)=A\left(a_{2}\right)=\{O, B\}$. Strategy spaces are $S_{\alpha_{0}}^{1}=\{\operatorname{Yes} O, \operatorname{Yes} B, \operatorname{No} O, \operatorname{No} B\}, S_{\alpha_{0}}^{2}=S_{\alpha_{1}}^{1}=S_{\alpha_{1}}^{2}=\{O, B\}$.

\subsubsection{Battle of the Sexes in Continuous Time}

The game is a Battle of the Sexes played in continuous time. As Anderson (1984) and Simon and Stinchcombe (1989) point out, the map from strategies to outcomes might not be well defined in continuous-time games, which implies that we cannot define the payoffs resulting from different strategy profiles. I will not spell out the details in Simon and Stinchcombe's (1989) model of continuous-time extensive-form games, I only present a simple example (that in fact falls within Simon and Stinchcombe's framework).

To avoid problems with the map from strategies to outcomes, I impose that players must switch infrequently from one action to the other. Time is indexed by $t \in[0,1]$. Assume that players choose either $O$ or $B$ at time $t=0$. Their decisions remain fixed for a period $\delta \in(0,1)$; at time $t=\delta$ they can choose to switch, represented by action 1 , or not to change their time 0 choice, represented by action 0 . An any posterior time $t$, players are allowed to choose 1 only if they have chosen 0 in $[t-\delta, t)$. That is, switches are irreversible for a length of time $\delta$. The players' "flow" payoffs are as in BoS in the introduction. If, at time $t$, they both choose $O$ then One gets a payoff of 2 while Two gets a payoff of 1 ; when they both choose $B$, then One gets 1 while Two gets 2 . If they choose different actions at time $t$ then they both get a payoff of 0 .

For any $t \in[0,1]$, the events until time $t$ are described by a pair of vectors $h_{t}=\left(h_{t}^{1}, h_{t}^{2}\right)$, with $h_{t}^{i}=\left(t_{1}^{i}, \ldots t_{k}^{i}\right)$ and where $t_{l}^{i}$ is the time of the $l$ th switch by player $i$. By the description above, we must have $t_{l}^{i}-t_{l-1}^{i} \geq \delta$ for $l=2, \ldots k$ and $t_{k}^{i} \leq t$. Player $i$ starts with action $\mathrm{O}$ if $t_{1}^{i}=0$, with action $\mathrm{B}$ if $t_{1}^{i}>0$. Any feasible $h_{t}$ is called a time- $t$ history. Let $H_{t}$ be the set of all time- $t$ histories.

The set of all information sets is $H=\cup_{t \in[0,1]} H_{t}$. Any history $h_{t}$ starts a subgame $H_{h_{t}}=\left\{h_{\tau} \in H: t \leq \tau, h_{\tau} \mid t=h_{t}\right\}$, where $h_{\tau} \mid t=h_{t}$ means that $h_{\tau}$ and $h_{t}$ coincide on 
switches before time $t$. For any history $h_{t}$, the actions available to player $i$ are $\{0,1\}$ or, if she has switched recently (so $\left.t-t_{k}<\delta\right),\{0\}$. I will show that the collection of

information sets is closed under increasing unions. Let $\left\{H_{h_{t}}: h_{t} \in \tilde{H}\right\}$ be an increasing collection of subgames and let $\underline{t}=\inf \left\{t: h_{t} \in \tilde{H}\right\}$. Then, all histories $h_{t}$ coincide on switches up to $\underline{t}$, let $h_{\underline{t}}$ be this common history. It is immediate that $H_{h_{\underline{t}}}=\cup_{h_{t} \in \tilde{H}} H_{h_{t}}$. Similarly, the collection of information sets is closed under intersections.

Strategies are maps $h_{t} \mapsto s^{i}\left(h_{t}\right) \in A^{i}\left(h_{t}\right)$, where $A^{i}\left(h_{t}\right)$ is $\{0\}$ if the last switch in $h_{t}$ was later than $t-\delta$ and $A^{i}\left(h_{t}\right)$ is $\{0,1\}$ else. A pair of strategies define, recursively, a finite collection of switches. A finite collection of switches gives, through the definition of flow payoffs above, the payoff associated to the strategy profile. Additivity of payoffs (from flow payoffs) implies immediately that payoffs are consistent.

\section{The Extended Best-Response Correspondence and Strategic Complementarities}

\subsection{Main Results}

In this paper I shall focus on subgame-perfect equilibria. In order to keep track of the best responses to opponents' strategies in each subgame, I need to introduce the set $\mathcal{S}^{i}=\left\{\mathrm{s}^{i} \in \Pi_{\alpha \in I} S_{\alpha}^{i}:\left.\mathrm{s}_{\alpha_{0}}^{i}\right|_{H_{\alpha}^{i}}=\mathrm{s}_{\alpha}^{i}\right\}$. This is the set of lists $\mathrm{s}^{i} \in \Pi_{\alpha \in I} S_{\alpha}^{i}$ so that the component $\mathrm{s}_{\alpha}^{i} \in S_{\alpha}^{i}$ that corresponds to subgame $H_{\alpha}$ coincides with the restriction of $\mathrm{s}_{\alpha_{0}}^{i} \in S_{\alpha_{0}}^{i}$-the strategy for the whole game-to subgame $H_{\alpha}$. Let $\mathcal{S}=\times_{i=1}^{n} \mathcal{S}^{i}$. For the example in Figure 2, recall that $S_{\alpha_{0}}^{1}=\left\{L L^{\prime}, L R^{\prime}, R L^{\prime}, R R^{\prime}\right\}, S_{\alpha_{1}}^{1}=\left\{L^{\prime}, R^{\prime}\right\}$, and $S_{\alpha_{2}}^{1}=\left\{L^{\prime}, R^{\prime}\right\}$. So,

$$
\mathcal{S}^{1}=\left\{\left(L L^{\prime}, L^{\prime}, L^{\prime}\right),\left(L R^{\prime}, R^{\prime}, R^{\prime}\right),\left(R L^{\prime}, L^{\prime}, L^{\prime}\right),\left(R R^{\prime}, R^{\prime}, R^{\prime}\right)\right\}
$$

which is really the same set as $S_{\alpha_{0}}^{1}$. In general $S^{i}$ and $\mathcal{S}^{i}$ are isomorphic: identify $\mathrm{s}^{i} \in \mathcal{S}^{i}$ with $\mathrm{s}_{\alpha_{0}}^{i} \in S^{i}$. In the rest of the paper I will frequently identify $S$ and $\mathcal{S}$.

Definition 4 Player $i$ 's extended best-response correspondence $\beta^{i}: \mathcal{S} \rightarrow \mathcal{S}^{i}$ is 
defined by:

$$
\beta^{i}(\mathrm{~s})=\left\{\mathrm{s}^{i} \in \mathcal{S}^{i}: u_{\alpha}^{i}\left(\mathrm{~s}_{\alpha}^{i}, \mathrm{~s}_{\alpha}^{-i}\right) \geq u_{\alpha}^{i}\left(\hat{s}_{\alpha}^{i}, \mathrm{~s}_{\alpha}^{-i}\right) \text { for all } \hat{s}_{\alpha}^{i} \in S_{\alpha}^{i} \text {, for all } \alpha \in I\right\} \text {. }
$$

The game's extended best-response correspondence is $\beta: \mathcal{S} \rightarrow \mathcal{S}$, defined as $\beta(\mathrm{s})=$ $\times_{i \in N} \beta_{i}(s)$.

Player $i$ 's extended best-response correspondence assigns a strategy that is a best response in each subgame to her opponents' strategy. A game $\Gamma$ 's SPNE can be analyzed by means of its extended best-response correspondence $\beta$. Lemma 1 shows the usefulness of the extended best-response construction. The construction of $\beta$ shows immediately why Lemma 1 is true, so the lemma's proof is omitted.

Lemma 1 The set of SPNE of a generalized extensive-form game equals the fixed points of its extended best-response correspondence.

Lemma 2 shows that $\beta$ is not a vacuous construction. The idea behind its proof is simple. Given opponents' strategies $s_{\alpha_{0}}^{-i}$, if $s_{\alpha_{0}}^{i}$ is a best response for player $i$ in the whole game $H_{\alpha_{0}}$, then $s_{\alpha_{0}}^{i}$ should prescribe a best response for subgames that are reached under $s_{\alpha_{0}}^{-i}$. Also, $i$ is indifferent between strategies on subgames that are not reached. Modifying $s_{\alpha_{0}}^{i}$ to play a best response to $s_{\alpha_{0}}^{-i}$ also on non-reached subgames yields, by consistency of payoffs, a strategy that is still a best response to $s_{\alpha_{0}}^{-i}$ in the original game $H_{\alpha_{0}}$. Repeating this operation "subgame by subgame" we can obtain an element in $\beta(\mathrm{s})$.

The reasoning "subgame by subgame" suggests a proof by induction. Even in simple games (like infinitely repeated bimatrix games) the set of subgames is uncountable, so a proof by induction is not possible. The proper tool turns out - expectedly - to be Zorn's Lemma.

Lemma 2 For all $\mathrm{s} \in \mathcal{S}, \beta(\mathrm{s})$ is not empty.

Proof: Let $\mathrm{s} \in \mathcal{S}$ and fix $i \in N$. For any $\alpha \in I$, let $\beta_{\alpha}^{i}\left(\mathrm{~s}^{-i}\right)=\operatorname{argmax}_{s^{i} \in S_{\alpha}^{i}} u_{\alpha}^{i}\left(s^{i}, \mathrm{~s}_{\alpha}^{-i}\right)$. Tychonoff's Theorem implies that $S_{\alpha}^{i}$ is compact, so $\beta_{\alpha}^{i}\left(\mathrm{~s}^{-i}\right)$ is nonempty because $S_{\alpha}^{i}$ 
is compact and payoffs are upper semi-continuous in the player's own strategy. Let $\Omega=\left\{\left(s_{\alpha}^{i}, H_{\alpha}^{i}\right): \alpha \in I, s_{\alpha}^{i} \in \beta_{\alpha}^{i}\left(\mathrm{~s}^{-i}\right)\right\}$ be the set of pairs of best responses and subgames. Order $\Omega$ by $\preceq$, where $\left(s_{\alpha^{\prime}}^{i}, H_{\alpha^{\prime}}^{i}\right) \preceq\left(s_{\alpha}^{i}, H_{\alpha}^{i}\right)$ if and only if $H_{\alpha^{\prime}}^{i} \subseteq H_{\alpha}^{i}$ and $\left.s_{\alpha}^{i}\right|_{H_{\alpha^{\prime}}^{i}}=s_{\alpha^{\prime}}^{i}$. It is immediate to verify that $\preceq$ is a partial order on $\Omega$.

Let $\tilde{\Omega} \subseteq \Omega$ be a linearly ordered subset of $\Omega$. Say $\tilde{I} \subseteq I$ is such that $\tilde{\Omega}=$ $\left\{\left(s_{\alpha}^{i}, H_{\alpha}^{i}\right) \in \Omega: \alpha \in \tilde{I}\right\}$. Let $\hat{H}=\cup_{\alpha \in \tilde{I}} H_{\alpha}^{i}$, since $\mathcal{H}^{i}$ is closed under increasing unions $\hat{H} \in \mathcal{H}^{i}$. Let $\gamma \in I$ satisfy $H_{\gamma}^{i}=\hat{H}$. For any $h \in H_{\gamma}$, there is $\alpha \in \tilde{I}$ such that $h \in H_{\alpha}$; construct $s_{\gamma}^{i} \in S_{\gamma}^{i}$ by setting $s_{\gamma}^{i}(h)=s_{\alpha}^{i}(h)$. Since $\tilde{\Omega}$ is linearly ordered, $s_{\gamma}^{i}$ is well defined.

I will show that $\left(H_{\gamma}^{i}, s_{\gamma}^{i}\right)$ is an upper bound on $\tilde{\Omega}$ in $\Omega$. Clearly, $H_{\alpha}^{i} \subseteq H_{\gamma}^{i}$ and $\left.s_{\gamma}^{i}\right|_{H_{\alpha}^{i}}=s_{\alpha}^{i}$ for all $\alpha \in \tilde{I}$. Let $\bar{s}_{\gamma}^{i} \in \beta_{\gamma}^{i}\left(\mathrm{~s}^{-i}\right)$ and let $\left\{s_{\gamma}^{i}(\alpha)\right\}_{\alpha \in \tilde{I}}$ be the net in $S_{\gamma}^{i}$ obtained by $\left.s_{\gamma}^{i}(\alpha)\right|_{H_{\alpha}^{i}}=\left.s_{\gamma}^{i}\right|_{H_{\alpha}^{i}},\left.s_{\gamma}^{i}(\alpha)\right|_{H_{\gamma}^{i} \backslash H_{\alpha}^{i}}=\left.\bar{s}_{\gamma}^{i}\right|_{H_{\gamma}^{i} \backslash H_{\alpha}^{i}}$, and directing $\tilde{I}$ by set inclusion. Note that $s_{\gamma}^{i}(\alpha) \rightarrow s_{\gamma}^{i}$ in the product topology. Fix any $\tilde{s}_{\gamma}^{i} \in S_{\gamma}^{i}$. Then $\bar{s}_{\gamma}^{i} \in \beta_{\gamma}^{i}\left(\mathrm{~s}^{-i}\right)$ implies that $u_{\gamma}^{i}\left(\tilde{s}_{\gamma}^{i}, \mathrm{~s}_{\gamma}^{-i}\right) \leq u_{\gamma}^{i}\left(\bar{s}_{\gamma}^{i}, \mathrm{~s}_{\gamma}^{-i}\right)$. Now, $\left.s_{\gamma}^{i}\right|_{H_{\alpha}^{i}}=s_{\alpha}^{i} \in \beta_{\alpha}^{i}\left(\mathrm{~s}^{-i}\right)$ implies that $u_{\alpha}^{i}\left(\left.\bar{s}_{\gamma}^{i}\right|_{H_{\alpha}^{i}}, \mathrm{~s}_{\alpha}^{-i}\right) \leq$ $u_{\alpha}^{i}\left(\left.s_{\gamma}^{i}\right|_{H_{\alpha}^{i}}, \mathrm{~s}_{\alpha}^{-i}\right)$. By consistency of payoffs, then, for any $\alpha \in \tilde{I}, u_{\gamma}^{i}\left(\tilde{s}_{\gamma}^{i}, \mathrm{~s}_{\gamma}^{-i}\right) \leq u_{\gamma}^{i}\left(s_{\gamma}^{i}(\alpha), \mathrm{s}_{\gamma}^{-i}\right)$. But then $u_{\gamma}^{i}\left(\tilde{s}_{\gamma}^{i}, \mathrm{~s}_{\gamma}^{-i}\right) \leq u_{\gamma}^{i}\left(s_{\gamma}^{i}, \mathrm{~s}_{\gamma}^{-i}\right)$, as $s_{\gamma}^{i}(\alpha) \rightarrow s_{\gamma}^{i}$ and payoffs are upper semi-continuous. This shows that $\left(H_{\gamma}^{i}, s_{\gamma}^{i}\right) \in \Omega$, so $\left(H_{\gamma}^{i}, s_{\gamma}^{i}\right)$ is an upper bound on $\tilde{\Omega}$.

The linearly ordered set $\tilde{\Omega}$ was arbitrary. By Zorn's lemma there is a maximal element, say $\left(H_{\alpha}^{* i}, s_{\alpha}^{* i}\right)$, of $\Omega$. Suppose $H_{\alpha}^{* i} \neq H_{\alpha_{0}}^{i}$. Let $\bar{s}_{\alpha_{0}}^{i} \in \beta_{\alpha_{0}}^{i}\left(\mathrm{~s}^{-i}\right)$. Define $s_{\alpha_{0}}^{i} \in S_{\alpha_{0}}^{i}$ by $\left.s_{\alpha_{0}}^{i}\right|_{H_{\alpha_{0}}^{i} \backslash H_{\alpha}^{* i}}=\left.\bar{s}_{\alpha_{0}}^{i}\right|_{H_{\alpha_{0}}^{i} \backslash H_{\alpha}^{* i}}$ and $\left.s_{\alpha_{0}}^{i}\right|_{H_{\alpha}^{* i}}=s_{\alpha}^{* i}$. Now, $\left(H_{\alpha}^{* i}, s_{\alpha}^{* i}\right) \in \Omega$ implies that $s_{\alpha}^{* i} \in \beta_{\alpha}^{i}\left(\mathrm{~s}^{-i}\right)$. Let $\tilde{s}_{\alpha_{0}}^{i} \in S_{\alpha_{0}}^{i}$, then $u_{\alpha_{0}}^{i}\left(\tilde{s}_{\alpha_{0}}^{i}, \mathrm{~s}_{\alpha_{0}}^{-i}\right) \leq u_{\alpha_{0}}^{i}\left(\bar{s}_{\alpha_{0}}^{i}, \mathrm{~s}_{\alpha_{0}}^{-i}\right)$ and $u_{\alpha}^{i}\left(\left.\bar{s}_{\alpha_{0}}^{i}\right|_{H_{\alpha}^{* i}}, \mathrm{~s}_{\alpha}^{-i}\right) \leq u_{\alpha}^{i}\left(\left.s_{\alpha_{0}}^{* i}\right|_{H_{\alpha}^{* i}}, \mathrm{~s}_{\alpha}^{-i}\right)$. By consistency of payoffs, $u_{\alpha_{0}}^{i}\left(\tilde{s}_{\alpha_{0}}^{i}, \mathrm{~s}_{\alpha_{0}}^{-i}\right) \leq u_{\alpha_{0}}^{i}\left(s_{\alpha_{0}}^{i}, \mathrm{~s}_{\alpha_{0}}^{-i}\right)$. Hence, $s_{\alpha_{0}}^{i} \in \beta_{\alpha_{0}}^{i}\left(\mathrm{~s}^{-i}\right)$, so that $\left(H_{\alpha_{0}}^{i}, s_{\alpha_{0}}^{i}\right) \in \Omega$ and $\left(H_{\alpha}^{* i}, s_{\alpha}^{* i}\right) \preceq\left(H_{\alpha_{0}}^{i}, s_{\alpha_{0}}^{i}\right)$. But $\left(H_{\alpha}^{* i}, s_{\alpha}^{* i}\right) \neq\left(H_{\alpha_{0}}^{i}, s_{\alpha_{0}}^{i}\right)$. Contradiction, since $\left(H_{\alpha}^{* i}, s_{\alpha}^{* i}\right)$ is maximal.

Construct $\mathrm{s}^{* i} \in \mathcal{S}^{i}$ by setting $\mathrm{s}_{\alpha}^{* i}=\left.s_{\alpha_{0}}^{* i}\right|_{H_{\alpha}^{i}}$ for all $\alpha \in I$. It is then immediate that $\mathrm{s}^{* i} \in \beta^{i}(\mathrm{~s})$ since, by construction, for all $\alpha \in I, \mathrm{~s}_{\alpha}^{* i}=s_{\alpha}^{* i} \in \beta_{\alpha}^{i}\left(\mathrm{~s}^{-i}\right)$.

Extended best-response correspondences translate the problem of finding SPNE to a fixed point problem. By adding the assumption of strategic complementarities, fixed points are obtained by a version of Tarski's Fixed Point Theorem and the sets of SPNE 
can be analyzed by "lattice programming" techniques. Potentially, though, extended best-response correspondences are useful in other classes of extensive-form games as well.

Theorem 1 If $\Gamma$ is an extensive-form game of strategic complementarities, then its SPNE form a non-empty, complete lattice.

Proof: I need to show that $\beta$ is monotone increasing in the strong set order and takes non-empty, closed values in order to apply Zhou's (1994) version of Tarski's fixed point theorem. First I show that $\beta$ is monotone increasing in the strong set order. Let $\mathbf{s}, \mathrm{z} \in \mathcal{S}$ with $\mathbf{s} \prec \mathbf{z}$. Let $\mathbf{s}^{\prime} \in \beta(\mathbf{s})$ and $\mathbf{z}^{\prime} \in \beta(\mathbf{z})$. By Theorem 4 in Milgrom and Shannon (1994), for every $\alpha \in I, \mathrm{~s}_{\alpha}^{\prime i} \vee \mathrm{z}_{\alpha}^{\prime i} \in \operatorname{argmax}_{s \in S_{\alpha}^{i}} u_{\alpha}^{i}\left(s, \mathrm{z}_{\alpha}^{-i}\right)$ and $\mathrm{s}_{\alpha}^{i} \wedge \mathrm{z}_{\alpha}^{\prime i} \in \operatorname{argmax}_{s \in S_{\alpha}^{i}} u_{i}^{\alpha}\left(s, \mathrm{~s}_{-i, \alpha}\right)$. Hence, $\mathbf{s}^{\prime} \vee z^{\prime} \in \beta(z)$ and $\mathbf{s}^{\prime} \wedge \mathbf{z}^{\prime} \in \beta(\mathrm{s})$, proving that $\beta$ is increasing in the strong set order.

That $\beta$ takes closed values is an immediate consequence of upper semi-continuity of payoffs in each subgame. By Lemma 2, $\beta$ takes non-empty values. Hence, by Zhou's version of Tarski's fixed point theorem, the set of fixed points of $\beta$ is a complete lattice. Lemma 1 implies that the set of SPNE is a complete lattice.

Theorem 1 implies that there is a smallest and a largest SPNE of any extensiveform game of strategic complementarities. Note that the subgames of any extensive-form game of strategic complements are also extensive-form games of strategic complements. By Theorem 1, then, each subgame has a smallest and a largest SPNE strategy profile. It turns out that the extremal SPNE of any subgame are obtained from the extremal SPNE of the whole game. ${ }^{5}$

The collection of subgames $\left\{H_{\alpha}: \alpha \in I\right\}$ is closed under intersections if for any $\alpha, \alpha^{\prime} \in I$, there is $\xi \in I$ such that $H_{\xi}=H_{\alpha} \cap H_{\alpha^{\prime}}$. Any well-defined game tree has subgames that are closed under intersections (in fact subgames are either nested or disjoint, so they are trivially closed under intersections).

Theorem 2 Let $\Gamma$ be an extensive-form game of strategic complementarities with subgames that are closed under intersections; let $\underline{s}$ be its smallest SPNE and $\bar{s}$ its largest

\footnotetext{
${ }^{5}$ This has important consequences. It can be seen that, in multi-stage games, the extremal equilibria are Markov-Perfect.
} 
SPNE. If $H_{\alpha}$ with $\alpha \in I$ is any subgame, then $\left.\underline{s}\right|_{H_{\alpha}}$ and $\left.\bar{s}\right|_{H_{\alpha}}$ are, respectively, the smallest and largest SPNE of the extensive-form game corresponding to $H_{\alpha}$.

Proof: Suppose, by way of contradiction, that there is a subgame $H_{\alpha}$ with a smallest SPNE strategy profile $s_{\alpha}$ that is not equal to $\left.\underline{s}\right|_{H_{\alpha}}$. Let $\tilde{\mathcal{S}}=\left\{\hat{\mathbf{s}} \in \mathcal{S}: \hat{\mathbf{s}}_{\alpha}=s_{\alpha}\right\}$, by repeating the arguments above we obtain that $\tilde{\mathcal{S}}$ is a complete lattice. Let $\tilde{\beta}: \tilde{\mathcal{S}} \rightarrow \tilde{\mathcal{S}}$ be defined by $\tilde{\beta}(\mathrm{s})=$ $\left\{\hat{\mathbf{s}} \in \tilde{\mathcal{S}}: u_{\gamma}^{i}\left(s_{\gamma}^{\prime i}, \mathbf{s}_{\gamma}^{-i}\right) \leq u_{\gamma}^{i}\left(\hat{\mathbf{s}}_{\gamma}^{i}, \mathbf{s}_{\gamma}^{-i}\right)\right.$ for all $s_{\gamma}^{\prime i} \in S_{\gamma}^{i},\left.s_{\gamma}^{\prime i}\right|_{H_{\alpha}}=s_{\alpha}^{i}, i \in N$, and $\left.\gamma \in I, H_{\gamma} \nsubseteq H_{\alpha}\right\}$.

That $\tilde{\beta}$ is monotone increasing in the strong set order and closed-valued follows from arguments similar to those proving that $\beta$ is monotone increasing and closed-valued. That $\tilde{\beta}$ has non-empty values can be proved by following the steps in the proof of Lemma 2 , and restricting the optimizing strategies to equal $s_{\alpha}^{i}$ on information sets that also belong to $H_{\alpha}$.

By Zhou's version of Tarski's Theorem, there is a fixed point $s \in \tilde{\mathcal{S}}$ of $\tilde{\beta}$. I claim that this is a SPNE of the whole game $H_{\alpha_{0}}$. Fix $i \in N$. For any $\gamma \in I$ with $H_{\gamma} \subseteq H_{\alpha}$, $\left.\mathbf{s}_{\alpha_{0}}\right|_{H_{\gamma}}=\left.s_{\alpha}\right|_{H_{\gamma}}$. But $\left.\mathbf{s}_{\alpha_{0}}^{i}\right|_{H_{\gamma}} \in \beta_{\gamma}^{i}(\mathrm{~s})$ since $H_{\gamma}$ is a subgame of $H_{\alpha}$ and $s_{\alpha}$ is a SPNE in $H_{\alpha}$. Let $\gamma \in I$ with $H_{\gamma} \nsubseteq H_{\alpha}$. $\Gamma$ has subgames that are closed under intersections, hence there is $\xi \in I$ with $H_{\xi}=H_{\gamma} \cap H_{\alpha}$. Since $H_{\xi}$ is a subgame of $H_{\alpha}, \mathbf{s}_{\xi}=\left.s_{\alpha}\right|_{H_{\xi}}$ so $\mathbf{s}_{\xi} \in \beta_{\xi}^{i}(\mathbf{s})$ because $s_{\alpha}$ is a SPNE in subgame $H_{\alpha}$. Then, $\mathbf{s}_{\gamma}^{i} \in \beta_{\gamma}^{i}(\mathbf{s})$ because if there is $\hat{s}_{\gamma}^{i} \in S_{\gamma}^{i}$ with $u_{\gamma}^{i}\left(\mathbf{s}_{\gamma}^{i}, \mathbf{s}_{\gamma}^{-i}\right)<u_{\gamma}^{i}\left(\hat{s}_{\gamma}^{i}, \mathbf{s}_{\gamma}^{-i}\right)$ it must be that $\hat{s}_{\gamma}^{i}$ and $\mathbf{s}_{\gamma}^{i}$ differ on the subgame $H_{\xi}=H_{\gamma} \cap H_{\alpha}$. But, using consistency of payoffs,

$$
u_{\gamma}^{i}\left(\mathbf{s}_{\gamma}^{i}, \mathbf{s}_{\gamma}^{-i}\right)<u_{\gamma}^{i}\left(\hat{s}_{\gamma}^{i}, \mathbf{s}_{\gamma}^{-i}\right) \leq u_{\gamma}^{i}\left(\left.\hat{s}_{\gamma}^{i}\right|_{H_{\gamma} \backslash H_{\alpha}}, \mathbf{s}_{\xi}^{i}, \mathbf{s}_{\gamma}^{-i}\right)
$$

impossible since $\mathbf{s} \in \tilde{\beta}(\mathrm{s})$. Hence, $\mathbf{s}_{\gamma}^{i} \in \beta_{\gamma}^{i}(\mathbf{s})$ for any $\gamma \in I$ so $\mathbf{s}$ is a SPNE.

By Theorem 1 there is a SPNE $\tilde{s}=\underline{s} \wedge s_{\alpha_{0}}$ because the set of SPNE is a lattice. Since $\underline{s}$ and $\mathbf{s}_{\alpha_{0}}$ differ on $H_{\alpha}, \tilde{s}$ is smaller than $\underline{s}$, a contradiction.

Once the structure of complementarities is present, comparative statics results for the extremal SPNE follow from well-known results in Monotone Comparative Statics. 


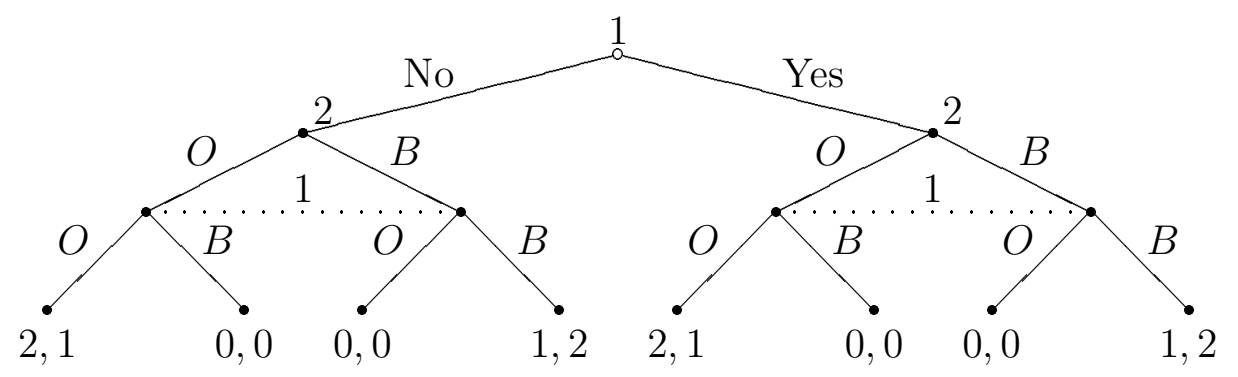

Figure 3: Optional BoS II

Definition 5 Let $T$ be a partially ordered set. The collection $\Gamma(t): t \in T$ is an increasing family of extensive-form games if, for any $t \in T, \Gamma(t)=\left\{N, H,\left\{H_{\alpha}: \alpha \in I\right\}\right.$, $\left.\left\{H_{i}: i \in N\right\},\left\{A_{t}(h): h \in H\right\},\left\{u_{\alpha t}^{i}: i \in N, \alpha \in I\right\}\right\}$ is an extensive-form game of strategic complementarities; if for all $h \in H, A_{t}(h)$ is increasing in the strong set order in $t$ and if, for all $i \in N \alpha \in I, u_{\alpha t}^{i}$ satisfies the single-crossing condition in $\left(s_{\alpha}^{i}, t\right)$.

Theorem 3 Let $\{\Gamma(t): t \in T\}$ be an increasing family of extensive-form games. Let $t, t^{\prime} \in T$ with $t \preceq t^{\prime}$. The smallest (largest) SPNE of $\Gamma(t)$ is smaller, as an element of $S$, than the smallest (largest) SPNE of $\Gamma\left(t^{\prime}\right)$.

Proof: Let $\beta_{t}$ and $\beta_{t^{\prime}}$ be the extended best-response correspondences of $\Gamma(t)$ and $\Gamma\left(t^{\prime}\right)$, respectively. An argument similar to the proof that the extended best-response function is monotone increasing in the proof of Theorem 1 establishes that, for any $\mathbf{s} \in \mathcal{S}, \beta_{t}(\mathbf{s})$ is smaller than $\beta_{t^{\prime}}(\mathrm{s})$ in the strong set order. The result then follows from Theorem 2.5.2 in Topkis (1998).

\section{Restrictiveness of Complementarities}

Optional BoS in the introduction is a game of strategic complementarities. Optional BoS II in Figure 3 cannot be made into a game of strategic complementarities. First, say that Yes is larger than No and repeat the argument from the introduction: An increase in One's strategy from No-OO to Yes-OO makes Two shift from $B$ to $O$ in the game following Yes. Strategic complementarities requires then that the action $O$ is larger than $B$ at this information set. But then, this gives a decreasing response when we shift 


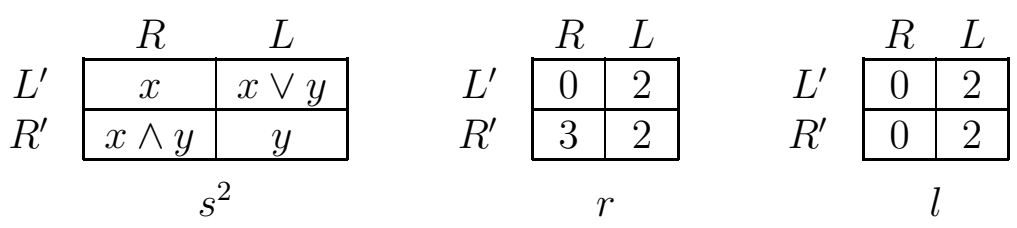

Figure 4: Payoffs to Player One in the game in Figure 2

from No- $O B$ to Yes- $O B$. The solution in the introduction was to change the order on $\{$ Yes, No\}. But here this clearly gives rise to the same problem in the subgame following No.

Since BoS is a game of strategic complementarities, Optional BoS II shows that the property of having complementarities is not robust to the addition of an irrelevant move. This is true also in normal-form games, not really the reason why complementarities are especially restrictive in extensive-form games.

More importantly, off the path specified by a strategy profile, players are indifferent between actions. This indifference makes the single-crossing property kick in. If the information set $h$ is off the path of a strategy profile $\left(s^{i}, s^{-i}\right)$ then player $i$ is indifferent between strategies that differ on $A(h)$. Now, if $s^{\prime-i}$ is a larger strategy, and $h$ is on the path of $\left(s^{i}, s^{\prime-i}\right)$, then it must be that payoffs are such that, if $a, a^{\prime} \in A(h)$ and $a$ is smaller than $a^{\prime}$, then $a$ is preferred to $a^{\prime}$. That is, preferences have to coincide with the order on actions for every strategy profile that has $h$ "on its path". This is the reason why complementarities are specially restrictive in extensive-form games. An open question is to characterize the set of extensive-form games with strategic complementarities. This has not been done for normal-form games, and it seems that the dynamic strategic interactions present special difficulties.

Finally, I will show that the game in Figure 2 is a game of strategic complementarities. Order the actions so that $L$ is larger than $R, l$ is larger than $r$ and $L^{\prime}$ is larger that $R^{\prime}$. Consider Figure 4. The two matrices to the right show the payoffs to player One for each of the eight possible strategy profiles in this game. The Figure is intended to illustrate the lattice structure on strategies. 
Fix Two's strategy $s^{2}=r$. We need to check that $s^{1} \mapsto u^{1}\left(s^{1}, r\right)$ is quasisupermodular. Here, $u^{1}\left(R L^{\prime}, r\right)=0<u^{1}\left(R L^{\prime} \wedge L R^{\prime}, r\right)=u^{1}\left(R R^{\prime}, r\right)=3$, so the requirement in the definition of quasisupermodularity is vacuous. Fix $s^{2}=l$, then $u^{1}\left(R L^{\prime} \wedge L R^{\prime}, l\right)=$ $u^{1}\left(R R^{\prime}, l\right)=0$ and $u^{1}\left(R L^{\prime}, l\right)=0$ so we need $u^{1}\left(L R^{\prime}, l\right) \leq u^{1}\left(R L^{\prime} \vee L R^{\prime}, l\right)$. Which is

satisfied since $u^{1}\left(R L^{\prime} \vee L R^{\prime}, l\right)=u^{1}\left(L L^{\prime}, l\right)=2$ and $u^{1}\left(L R^{\prime}, l\right)=2$. Now, we need to check that the players' payoffs satisfy the single-crossing property. Note from Figure 4 that, when $s^{2}=r$, no increase in a strategy by player One is profitable. Since $r<l$ the single-crossing property in player One's payoffs is satisfied vacuously.

$$
\begin{array}{c|c|c|}
\multicolumn{1}{c}{} & \multicolumn{1}{c}{R} & \multicolumn{1}{c}{L} \\
L^{\prime} & 0 & 0 \\
\cline { 2 - 3 } R^{\prime} & -1 & 0 \\
\cline { 2 - 3 } & \multicolumn{3}{c}{s^{2}}
\end{array}
$$

Figure 5: Gain from increasing Two's strategy: $u^{2}\left(s^{1}, l\right)-u^{2}\left(s^{1}, r\right)$.

To see that Two's payoffs satisfy the single-crossing property, consider Figure 5. The figure shows the gain to Two $u^{2}\left(s^{1}, l\right)-u^{2}\left(s^{1}, r\right)$ from increasing his strategy from $r$ to $l$. It is seen directly from the figure that whenever $u^{2}\left(s^{1}, l\right)-u^{2}\left(s^{1}, r\right) \geq 0$ and $s^{1}<s^{1 \prime}$ then $u^{2}\left(s^{1 \prime}, l\right)-u^{2}\left(s^{1 \prime}, r\right) \geq 0$. This establishes that the game in Figure 2 is a game of strategic complementarities.

\section{References}

Anderson, R. M. (1984): "Quick Response Equilibria," UC Berkeley C.R.M. I.P. \# 323 .

FudenberG, D., And D. Levine (1983): "Subgame-Perfect Equilibria of Finite- and Infinite-Horizon Games," Journal of Economic Theory, 31(1), 251-268.

FudenberG, D., And J. Tirole (1991): Game Theory. MIT Press.

HARris, C. (1985a): "A Characterization of the Perfect Equilibria of Infinite Horizon Games," Journal of Economic Theory, 37(1), 99-125. 
(1985b): "Existence and Characterization of Perfect Equilibrium in Games of Perfect Information," Econometrica, 53(3), 613-628.

Harris, C., P. Reny, and A. Robson (1995): "The Existence of Subgame-Perfect Equilibrium in Continuous Games with Almost Perfect Information: A Case for Public Randomization," Econometrica, 63(3), 507-544.

Hellwig, M., And W. Leininger (1987): "On The Existence of Subgame-Perfect Equilibrium in Infinite-Action Games of Perfect Information," Journal of Economic Theory, 43(1), 55-75.

Milgrom, P., and J. Roberts (1990): "Rationalizability, Learning and Equilibrium in Games with Strategic Complementarities," Econometrica, 58(6), 1255-1277.

Milgrom, P., And C. Shannon (1994): "Monotone Comparative Statics," Econometrica, 62(1), 157-180.

Osborne, M. J., And A. Rubinstein (1994): A Course in Game Theory. Mit Press.

Simon, L. K., and M. B. Stinchcombe (1989): "Extensive Form Games in Continuous Time: Pure Strategies," Econometrica, 57(5), 1171-1214.

Topkis, D. M. (1979): "Equilibrium Points in Nonzero-Sum n-Person Submodular Games," SIAM Journal of Control and Optimization, 17(6), 773-787.

— (1998): Supermodularity and Complementarity. Princeton University Press.

Vives, X. (1990): "Nash Equilibrium with Strategic Complementarities," Journal of Mathematical Economics, 19(3), 305-321.

_ (1999): Oligopoly Pricing. MIT Press.

Zhou, L. (1994): "The Set of Nash Equilibria of a Supermodular Game Is a Complete Lattice," Games and Economic Behavior, 7(2), 295-300. 